\title{
Maps preserving zero products
}

by

\author{
J. Alaminos (Granada), M. BrešAr (Ljubljana and Maribor), \\ J. Extremera (Granada), and A. R. Villena (Granada)
}

\begin{abstract}
A linear map $T$ from a Banach algebra $A$ into another $B$ preserves zero products if $T(a) T(b)=0$ whenever $a, b \in A$ are such that $a b=0$. This paper is mainly concerned with the question of whether every continuous linear surjective map $T: A \rightarrow B$ that preserves zero products is a weighted homomorphism. We show that this is indeed the case for a large class of Banach algebras which includes group algebras.

Our method involves continuous bilinear maps $\phi: A \times A \rightarrow X$ (for some Banach space $X$ ) with the property that $\phi(a, b)=0$ whenever $a, b \in A$ are such that $a b=0$. We prove that such a map necessarily satisfies $\phi(a \mu, b)=\phi(a, \mu b)$ for all $a, b \in A$ and for all $\mu$ from the closure with respect to the strong operator topology of the subalgebra of $\mathcal{M}(A)$ (the multiplier algebra of $A$ ) generated by doubly power-bounded elements of $\mathcal{M}(A)$. This method is also shown to be useful for characterizing derivations through the zero products.
\end{abstract}

Introduction. S. Banach [4] was the first to describe isometries on $L^{p}([0,1])$ with $p \neq 2$. Although Banach did not give the full proof for this case (this was provided by J. Lamperti [26]), he made the key observation that isometries on $L^{p}([0,1])$ must take functions with disjoint support into functions with disjoint support. This property arises in a variety of situations and has been considered by several authors. For example, in the theory of Banach lattices there is an extensive literature about linear maps $T: X \rightarrow Y$, where $X$ and $Y$ are Banach lattices, with the property that $|T(x)| \wedge|T(y)|=0$ whenever $x, y \in X$ are such that $|x| \wedge|y|=0$. Such maps are usually called disjointness preserving operators or d-homomorphisms. The reader interested in this setting is referred to the monograph [1]. The concept of a disjointness preserving operator was exported to function algebras by E. Beckenstein and L. Narici (see [5] for general information).

2000 Mathematics Subject Classification: 47B47, 47B48, 43A20, 43A22, 46L05.

Key words and phrases: group algebra, $C^{*}$-algebra, homomorphism, weighted homomorphism, derivation, generalized derivation, separating map, disjointness preserving map, zero product preserving map, doubly power-bounded element. 
Let $A$ and $B$ be function algebras. Linear operators $T: A \rightarrow B$ with the property that $T(a) T(b)=0$ whenever $a, b \in A$ are such that $a b=0$ have been studied over the years and by many authors. They are usually considered under the name Lamperti operators or separating maps. Typical examples are provided by weighted composition operators, and it turns out that in certain important cases these are the canonical continuous separating maps. The concept of separating maps can be extended in various ways to general (possibly noncommutative) Banach algebras, and in fact even to pure algebras. The most common and natural way is to consider literally the same condition, that is,

$$
a, b \in A, a b=0 \Rightarrow T(a) T(b)=0,
$$

where $T$ is a linear map between algebras $A$ and $B$. Usually such operators are called zero product preserving maps and the standard goal is to characterize them as weighted homomorphisms. For recent results on such and related maps defined on operator algebras we refer the reader to $[2,3$, $9-13,20,21,25,30,35]$, and on the other hand to [17] where group algebras corresponding to locally compact abelian groups are considered.

Our method for investigating a continuous linear map $T: A \rightarrow B$ preserving zero products consists in introducing a continuous bilinear map $\phi: A \times A \rightarrow B$ by $\phi(a, b)=T(a) T(b)(a, b \in A)$ which obviously satisfies the condition $\phi(a, b)=0$ whenever $a, b \in A$ are such that $a b=0$. Motivated by this fact we study bilinear maps $\phi: A \times A \rightarrow X$ into some Banach space $X$ that preserve zero products in the sense that

$$
a, b \in A, a b=0 \Rightarrow \phi(a, b)=0 .
$$

The study of (0.2) was initiated in our preceding paper [2]. However, [2, Lemma 2.1] considers (0.2) only in a very special setting and the conclusion is much less general than the one that will be obtained in this paper.

The paper is organized as follows. In the first section we introduce the notation and terminology, and then the class of faithful Banach algebras $A$ satisfying the following condition:

$$
\text { (A) } A \subset \overline{\mathcal{D}(A)}{ }^{\text {so }} \text {. }
$$

Here $\mathcal{D}(A)$ stands for the subalgebra of the multiplier algebra of $A$ generated by the doubly power-bounded elements and $\overline{(\cdot)^{\text {so }}}$ denotes the closure with respect to the strong operator topology. We show that the class of Banach algebras having the property $(\mathbb{A})$ is really large, it includes: group algebras, $C^{*}$-algebras, and Banach algebras generated by idempotents.

Section 2 is concerned with continuous bilinear maps preserving zero products in the sense of (0.2). At the beginning we introduce another class of Banach algebras, namely those that have the following property: 
$(\mathbb{B})$ for any Banach space $X$ and every continuous bilinear map $\phi: A \times A$ $\rightarrow X,(0.2)$ implies that $\phi(a b, c)=\phi(a, b c)$ for all $a, b, c \in A$.

Under mild technical assumptions, the condition $\phi(a b, c)=\phi(a, b c)$ further implies that $\phi$ is of the form

$$
\phi(a, b)=\Phi(a b)
$$

for some continuous linear map $\Phi: A \rightarrow X$, which is clearly the best possible description of a map satisfying (0.2). For example, if $A$ is unital, then just take $b=\mathbf{1}$ and note that $\Phi$ can be defined according to $\Phi(a)=\phi(a, \mathbf{1})$. However, except in a few situations, throughout the paper we try to avoid the assumption that our algebras are unital, in order to cover some important examples of Banach algebras such as group algebras. On the other hand, we will often assume the existence of an approximate identity.

We show that the property $(\mathbb{B})$ is stable under the usual methods of constructing Banach algebras. However, it does not seem obvious why Banach algebras with the property $(\mathbb{B})$ should exist at all. A crucial result of the paper is Theorem 2.11 which tells us that for every Banach algebra $A$ we have:

$$
A \text { has the property }(\mathbb{A}) \Rightarrow A \text { has the property }(\mathbb{B}) .
$$

More precisely, Theorem 2.11 states that $(0.2)$ implies that $\phi(a \mu, b)=$ $\phi(a, \mu b)$ for all $a, b \in A$ and all $\mu \in \overline{\mathcal{D}}(A)^{\text {so }}$, from which (0.3) clearly follows. As is evident from this statement, our approach to zero product preservers is based on some special invertible elements (typical examples are point mass measures in the context of group algebras and unitary elements in $C^{*}$-algebras). This seems to be a novelty. The traditional approach is based on some other types of elements, particularly on idempotents. We also remark that the proof rests heavily on a technical result (Lemma 2.10) about $\ell^{1}(\mathbb{Z})$ using classical Fourier analysis.

Of course (0.3) gives a convincing justification for dealing with the class of Banach algebras with the property $(\mathbb{B})$. The part of the paper after Theorem 2.11 is mostly concerned with this class.

In the third section we consider the central theme of the paper, that is, continuous linear maps $T: A \rightarrow B$ between Banach algebras $A$ and $B$ that satisfy $T(a) T(b)=0$ whenever $a, b \in A$ are such that $a b=0$. Roughly speaking, the goal is to show that $T$ is close to a homomorphism. The heart of the paper are Theorems 3.4 and 3.8 which essentially say that under rather mild assumptions on Banach algebras $A$ and $B$, a continuous linear map $T: A \rightarrow B$ that preserves zero products is a weighted homomorphism, whenever $A$ has the property $(\mathbb{B})$. As a matter of fact, we show that if $G_{1}$ and $G_{2}$ are locally compact groups and $T: L^{1}\left(G_{1}\right) \rightarrow L^{1}\left(G_{2}\right)$ is a continuous surjective linear map preserving zero products, then there are an invertible 
measure $\mu$ in the centre of the measure algebra $M\left(G_{2}\right)$ and a continuous epimorphism $\Phi: L^{1}\left(G_{1}\right) \rightarrow L^{1}\left(G_{2}\right)$ with $T=\mu \Phi$.

Finally, in Section 4 we show that the study of bilinear maps preserving zero products contained in the second section is also useful for characterizing derivations through zero products. Over the last years a considerable attention has been paid to characterizations of such kind [2, 8, 9, 23, 37]. In the recent papers $[2,8]$, it was observed that characterizing derivations through the zero product analysis can be a powerful tool in studying local derivations, which is another popular research area.

1. The seminal property. The purpose of this section is to introduce the notation and terminology, and then the class of Banach algebras with the property $(\mathbb{A})$. We are actually mostly interested in another class of Banach algebras, namely those with the property $(\mathbb{B})$. Nevertheless, the property $(\mathbb{A})$ is of extreme importance for this paper because of its close connection with the property $(\mathbb{B})$.

1.1. Some notation and terminology. All Banach spaces and Banach algebras which we consider throughout this paper are assumed to be complex.

Let $X$ be a linear space. The linear span of a nonempty subset $S \subset X$ is denoted by $\operatorname{lin} S$. The algebra of linear operators from $X$ into itself is denoted by $\mathcal{L}(X)$. Let $\mathcal{B}(X)$ denote the Banach algebra of all continuous linear operators on a given nonzero complex Banach space $X$, and let $X^{*}$ denote the topological dual space of $X$. We write $\langle\cdot, \cdot\rangle$ for the dual pairing of $X$ and $X^{*}$.

Let $A$ be an algebra. Then:

(i) $A$ is left faithful if $\{a \in A: a A=\{0\}\}=\{0\}$;

(ii) $A$ is right faithful if $\{a \in A: A a=\{0\}\}=\{0\}$;

(iii) $A$ is faithful if $\{a \in A: a A=A a=\{0\}\}=\{0\}$.

We write $A^{\text {op }}$ for the opposite algebra to $A$. For a nonempty subset $S \subset A$ and $n \in \mathbb{N}$, set

$$
S^{n}=\operatorname{lin}\left\{a_{1} \cdots a_{n}: a_{1}, \ldots, a_{n} \in S\right\} .
$$

Further, alg $S$ denotes the subalgebra of $A$ generated by $S$.

Let $A$ be a Banach algebra. Then:

(i) A factors weakly if $A=A^{2}$;

(ii) $A$ is essential if $A=\overline{A^{2}}$.

A left [right] approximate identity for $A$ is a net $\left(\rho_{i}\right)_{i \in I}$ in $A$ such that $\lim \rho_{i} a=a\left[\lim a \rho_{i}=a\right]$ for each $a \in A$. An approximate identity for $A$ is a net $\left(\rho_{i}\right)_{i \in I}$ which is both a left and a right approximate identity. A [left or right] approximate identity $\left(\rho_{i}\right)_{i \in I}$ is bounded if $\sup _{i \in I}\left\|\rho_{i}\right\|<\infty$. It should be pointed out that if a Banach algebra has a bounded left approximate 
identity and a bounded right approximate identity, then it has a bounded approximate identity [14, Proposition 2.9.3]. In general, the existence of both a left approximate identity and a right approximate identity in a Banach algebra does not imply the existence of an approximate identity [15, §2]. Obviously, if $A$ has a left approximate identity, then $A$ is right faithful and essential. According to Cohen's factorization theorem [14, Theorem 2.9.24], if $A$ has a bounded left approximate identity, then $A$ factors weakly, in fact $A=\{a b: a, b \in A\}$.

Since approximate identities will play an important role in this paper, it seems appropriate to mention some relevant examples of Banach algebras containing them. For a more complete account we refer the reader to $[14,15]$, where the corresponding hereditary properties are also studied. We shall mention just a few concrete examples and a few classes of algebras in which we are mostly interested in this paper (see remarks concerning the conditions $(\mathbb{A})$ and $(\mathbb{B})$ below).

- Let $G$ be a locally compact group with left Haar measure $\lambda$. As is customary, we write $L^{1}(G)$ for the Banach algebra of those (equivalence classes of) Borel functions on $G$ which are integrable with respect to $\lambda$. The group algebra $L^{1}(G)$ has a bounded approximate identity of bound 1 [14, Theorem 3.3.23].

- Every $C^{*}$-algebra has a bounded approximate identity of bound 1 [14, Theorem 3.2.21].

- The Schatten class $S_{p}(H)$ has an approximate identity for every Hilbert space $H$ and $1 \leq p<\infty$.

- If $X$ is a Banach space with the approximation property, then $\mathcal{A}(X)$, the algebra of all approximable operators, has a left approximate identity [32, Proposition 3.1.1]. Furthermore, $\mathcal{A}(X)$ has a bounded left approximate identity if and only if the Banach space $X$ has the bounded approximation property [32, Corollary 3.1.3]. The usual examples of separable Banach spaces have the bounded approximation property and, as a matter of fact, they even have a basis. The space $L^{p}(\mu)$ for any measure space $(\Omega, \Sigma, \mu)$ and $1 \leq p \leq \infty$ and the space $C_{0}(\Omega)$ for any locally compact Hausdorff space have the bounded approximation property (see [32, Definition 3.1.8 and Examples 3.1.12]).

1.2. Introducing the property $(\mathbb{A})$. Let $A$ be an algebra. A multiplier on $A$ is a pair $(L, R)$, where $L, R: A \rightarrow A$ are linear maps such that, for all $a, b \in A$, the following identities hold:

$$
L(a b)=L(a) b, \quad R(a b)=a R(b), \quad \text { and } \quad a L(b)=R(a) b .
$$

The set of all multipliers on $A$ is denoted by $\mathcal{M}(A)$. It is a unital subalgebra of $\mathcal{L}(A) \times \mathcal{L}(A)^{\text {op }}$ and it is called the multiplier algebra of $A$. The map 


$$
a \mapsto\left(L_{a}, R_{a}\right), \quad A \rightarrow \mathcal{M}(A),
$$

where $L_{a}(b)=a b=R_{b}(a)$ for all $a, b \in A$, is a homomorphism, and it is a monomorphism if and only if $A$ is faithful. In this case we shall always identify $A$ with its image via this embedding and it is easily checked that $A$ is a two-sided ideal of $\mathcal{M}(A)$. Moreover, $A$ is unital if and only if $A=\mathcal{M}(A)$. As usual, the unitisation $A_{1}$ of $A$ is the subalgebra of $\mathcal{M}(A)$ generated by $A$ and $\mathbf{1 .}$

We now turn to the case when $A$ is a faithful Banach algebra. Then every multiplier on $A$ consists of continuous linear operators on $A$ so that $\mathcal{M}(A)$ becomes a unital closed subalgebra of $\mathcal{B}(A) \times \mathcal{B}(A)^{\mathrm{op}}$ and the embedding of $A$ into $\mathcal{M}(A)$ is continuous [14, Proposition 2.5.12]. An invertible element $\mu \in \mathcal{M}(A)$ is said to be doubly power-bounded if $\sup _{k \in \mathbb{Z}}\left\|\mu^{k}\right\|<\infty$. Faithful Banach algebras generated by the doubly power-bounded elements in the multiplier algebra will play the crucial role throughout this paper. Let $\mathcal{D}(A)$ stand for the subalgebra of $\mathcal{M}(A)$ generated by the doubly power-bounded elements in $\mathcal{M}(A)$. The strong operator topology on $\mathcal{M}(A)$, denoted by "so", is determined by the family of seminorms $\left\{p_{a}: a \in A\right\}$, where

$$
p_{a}(L, R)=\max \{\|L(a)\|,\|R(a)\|\} \quad((L, R) \in \mathcal{M}(A)) .
$$

Therefore, a net $\left(\mu_{i}\right)_{i \in I}$ in $\mathcal{M}(A)$ converges to $\mu \in \mathcal{M}(A)$ with respect to the strong operator topology if and only if $\lim \mu_{i} a=\mu a$ and $\lim a \mu_{i}=a \mu$ with respect to the norm for each $a \in A$.

Definition 1.1. We say that a Banach algebra $A$ has the property $(\mathbb{A})$ if it is faithful and satisfies

$$
A \subset \overline{\mathcal{D}(A)}{ }^{\text {so }} .
$$

Further, we say that a faithful Banach algebra $A$ has the property $\left(\mathbb{A}_{\mathbf{1}}\right)$ if

$$
A_{\mathbf{1}}=\overline{\operatorname{alg}\left\{u \in A_{\mathbf{1}}: u \text { is doubly power-bounded }\right\}} .
$$

Of course, $\left(\mathbb{A}_{\mathbf{1}}\right)$ is stronger than $(\mathbb{A})$.

REMARK 1.2. There are several important classes of elements in the multiplier algebra $\mathcal{M}(A)$ of a Banach algebra $A$ which lie in the closed algebra generated by doubly power-bounded elements.

(i) Let $A$ be a unital Banach algebra. For $a \in A$, we define the numerical range of $a$ by

$$
V(a)=\left\{\langle a, f\rangle: f \in A^{*},\langle\mathbf{1}, f\rangle=\|f\|=1\right\} ;
$$

this is a compact convex subset of the complex plane containing the spectrum of $a$. An element $a \in A$ is called hermitian if $V(a) \subset \mathbb{R}$, which is equivalent to the property that $\|\exp (i t a)\|=1$ for each $t \in \mathbb{R}($ see $[6, \S 10])$. It is well known that hermitian elements of a 
unital $C^{*}$-algebra are precisely the self-adjoint elements [6, Proposition 20 in $\S 12]$.

It should be pointed out that if $A$ is a Banach algebra and $\mu \in \mathcal{M}(A)$ is hermitian, then

$$
\mu=\frac{1}{i} \lim _{t \rightarrow 0} \frac{\exp (i t \mu)-\mathbf{1}}{t} \in \overline{\mathcal{D}(A)} .
$$

(ii) Throughout, the set of idempotents of a given algebra $A$ is denoted by $\mathcal{I}(A)$. Let $A$ be a Banach algebra and let $e \in \mathcal{I}(\mathcal{M}(A))$. Then $(\mathbf{1}-2 e)^{2}=\mathbf{1}$, which obviously implies that $\mathbf{1}-2 e$ is doubly powerbounded. Moreover, $e=\frac{1}{2} \mathbf{1}-\frac{1}{2}(\mathbf{1}-2 e)$ and hence $e \in \mathcal{D}(A)$.

1.3. Examples of Banach algebras with the property $(\mathbb{A})$. The goal of this short section is to collect some basic examples of Banach algebras which have the property $(\mathbb{A})$.

1. Group algebras. Let $G$ be a locally compact group. We write $M(G)$ for the Banach algebra of all complex, regular Borel measures on $G$. Of course, $L^{1}(G)$ can be thought of as the two-sided closed ideal of $M(G)$ consisting of all measures in $M(G)$ which are absolutely continuous with respect to $\lambda$. In fact, by Wendel's theorem [14, Theorem 3.3.40], $M(G)$ is nothing other than the multiplier algebra of $L^{1}(G)$. For every $t \in G$, the unit point mass measure $\delta_{t}$ at $t$ is clearly doubly power-bounded. Moreover, [14, Proposition 3.3.41] shows that $\left.\overline{\operatorname{lin}\left\{\delta_{t}: t \in G\right\}}\right\}^{\text {so }}=M(G)$. Hence $L^{1}(G)$ has the property (A).

2. $C^{*}$-algebras. Let $A$ be a $C^{*}$-algebra. Then $A_{1}$ becomes a unital $C^{*}$ algebra (see [14, Proposition 3.2.39]). It is well known that every element in $A_{1}$ is a linear combination of four unitary elements [34, Proposition I.4.9]; recall that an element $u \in A_{1}$ is unitary if $u u^{*}=u^{*} u=1$. Since unitary elements are clearly doubly power-bounded, $A_{\mathbf{1}}$ is generated by the doubly power-bounded elements and so $A$ has the property $\left(\mathbb{A}_{\mathbf{1}}\right)$.

3. Banach algebras generated by idempotents. In view of Remark 1.2(ii), if $A$ is a Banach algebra with the property that $A=\overline{\operatorname{alg} \mathcal{I}(A)}$, then $A$ has the property $\left(\mathbb{A}_{\mathbf{1}}\right)$. Some examples of such Banach algebras are the following.

(1) The Banach algebras $\mathcal{A}(X)$ and $\mathcal{N}(X)$ of all approximable operators and all nuclear operators, respectively, on any Banach space $X$.

(2) The classical Schatten class $S_{p}(H)$ for any Hilbert space $H$ and $1 \leq$ $p \leq \infty$.

(3) The Banach algebras $\ell^{p}(I)$ with $1 \leq p<\infty$ and $c_{0}(I)$ for any nonempty set $I$.

(4) The Banach algebras $L^{p}(G)$ with $1 \leq p<\infty$ and $C(G)$ (with convolution product) for any compact group $G$. Indeed, on account of [19, Theorem 27.21], the linear space $\mathcal{T}(G)$ of all trigonometric polynomials on $G$ (see [19, Definition 27.7]) is the linear span of the 
idempotents, and [16, Theorem 5.11] shows that $\mathcal{T}(G)$ is dense in $L^{p}(G)$ for $1 \leq p<\infty$ and $C(G)$.

(5) Topologically simple Banach algebras containing a nontrivial idempotent. Indeed, a nontrivial idempotent $e$ (i.e., $e \neq 0$ and $e \neq \mathbf{1}$ ) in such an algebra $A$ clearly cannot be contained in the centre of $A$. This implies that $\operatorname{alg} \mathcal{I}(A)$ contains a nonzero ideal of $A[8$, Lemma 2.1], from which $A=\overline{\operatorname{alg} \mathcal{I}(A)}$ follows.

\section{Bilinear maps preserving zero products}

Definition 2.1. Let $A$ be a Banach algebra and let $\phi: A \times A \rightarrow X$, where $X$ is a Banach space, be a continuous bilinear map. We say that $\phi$ preserves zero products if

$$
a, b \in A, a b=0 \Rightarrow \phi(a, b)=0 .
$$

2.1. Introducing the property $(\mathbb{B})$

Definition 2.2. A Banach algebra $A$ has the property $(\mathbb{B})$ if every continuous bilinear map $\phi: A \times A \rightarrow X$, where $X$ is an arbitrary Banach space, preserving zero products satisfies

$$
\phi(a b, c)=\phi(a, b c) \quad(a, b, c \in A) .
$$

The basic result in this section is Theorem 2.11 which shows that the class of Banach algebras with the property $(\mathbb{B})$ is large since it contains the class of algebras with the property $(\mathbb{A})$.

A typical example of a map $\phi$ from the above definition is the one given by $\phi(a, b)=\Phi(a b)$ where $\Phi: A \rightarrow X$ is a linear operator. Under mild assumptions this is also the only possible example.

Lemma 2.3. Let $A$ be a Banach algebra with the property $(\mathbb{B})$. If $A$ has a left approximate identity, then every continuous bilinear map $\phi: A \times A \rightarrow X$ preserving zero products is of the form

$$
\phi(a, b)=\Phi(a b) \quad(a, b \in A)
$$

for some linear operator $\Phi: A^{2} \rightarrow X$. Moreover, if $A$ has a bounded left approximate identity, then $\Phi$ is continuous.

Proof. Let $\left(\rho_{i}\right)_{i \in I}$ be a left approximate identity of $A$. Since $A$ has the property $(\mathbb{B})$, for all $a, b \in A$ we have

$$
\phi(a, b)=\lim _{i \in I} \phi\left(\rho_{i} a, b\right)=\lim _{i \in I} \phi\left(\rho_{i}, a b\right) .
$$

Therefore the net $\left(\phi\left(\rho_{i}, a\right)\right)_{i \in I}$ is convergent for each $a \in A^{2}$ and so we may define a linear operator

$$
\Phi: A^{2} \rightarrow X, \quad \Phi(a)=\lim _{i \in I} \phi\left(\rho_{i}, a\right) \quad\left(a \in A^{2}\right) .
$$


According to the definition of $\Phi$ and in view of $(2.1)$, we have $\phi(a, b)=\Phi(a b)$ for all $a, b \in A$.

If $\left(\rho_{i}\right)_{i \in I}$ is bounded, then, for every $a \in A$,

$$
\left\|\phi\left(\rho_{i}, a\right)\right\| \leq\|\phi\| \sup _{i \in I}\left\|\rho_{i}\right\|\|a\| \quad(i \in I)
$$

and so $\Phi$ is continuous with $\|\Phi\| \leq\|\phi\| \sup _{i \in I}\left\|\rho_{i}\right\|$.

Corollary 2.4. Let $A$ be a commutative Banach algebra with the property $(\mathbb{B})$. If $A$ has a left approximate identity, then every continuous bilinear map $\phi: A \times A \rightarrow X$ preserving zero products is symmetric, that is, $\phi(a, b)=\phi(b, a)$ for all $a, b \in A$.

Proof. By Lemma 2.3 we have $\phi(a, b)=\Phi(a b)=\Phi(b a)=\phi(b, a)$.

2.2. The heredity of the property $(\mathbb{B})$

Proposition 2.5. Let $A$ be a Banach algebra with the property $(\mathbb{B})$ and let $I$ be a closed two-sided ideal of $A$. Then the following assertions hold:

(i) $A / I$ has the property $(\mathbb{B})$.

(ii) If $\overline{\operatorname{lin}(A I)}=\overline{\operatorname{lin}(I A)}=I$ (in particular, if either $A$ or $I$ has an approximate identity), then $I$ has the property $(\mathbb{B})$.

Proof. Let $X$ be a Banach space. Let $\phi: A / I \times A / I \rightarrow X$ be a continuous linear map preserving zero products for some Banach space $X$. We then define $\psi: A \times A \rightarrow X$ by $\psi(a, b)=\phi(a+I, b+I)(a, b \in A)$, which preserves zero products, and so the property $(\mathbb{B})$ gives $\psi(a b, c)=\psi(a, b c)$ for all $a, b, c$ $\in A$. This clearly forces $\phi(x y, z)=\phi(x, y z)$ for all $x, y, z \in A / I$.

We now take a continuous bilinear map $\phi: I \times I \rightarrow X$ preserving zero products. Pick $u, v \in I$ and define $\psi_{u, v}: A \times A \rightarrow X$ by $\psi_{u, v}(a, b)=\phi(u a, b v)$ for all $a, b \in A$. We see that $\psi_{u, v}$ preserves zero products and so our assumption implies that $\psi_{u, v}(a b, c)=\psi_{u, v}(a, b c)$, and hence $\phi(u a b, c v)=\phi(u a, b c v)$ for all $a, b, c \in A$. Using $\overline{\operatorname{lin}(A I)}=\overline{\operatorname{lin}(I A)}=I$ we arrive at the required identity for $\phi$.

Proposition 2.6. Let $A$ be a Banach algebra with the property $(\mathbb{B})$, let $B$ be a Banach algebra, and let $\Phi: A \rightarrow B$ be a continuous homomorphism with dense range. Then $B$ has the property $(\mathbb{B})$.

Proof. Let $\psi: B \times B \rightarrow X$ be a continuous bilinear map preserving zero products, for some Banach space $X$. We then define $\phi: A \times A \rightarrow X$ by $\phi(a, b)=\psi(\Phi(a), \Phi(b))$ for all $a, b \in A$. It is clear that $\phi$ is a continuous bilinear map preserving zero products and so $\phi(a b, c)=\phi(a, b c)$ for all $a, b, c$ $\in A$. This can be rewritten as $\psi(\Phi(a) \Phi(b), \Phi(c))=\psi(\Phi(a), \Phi(b) \Phi(c))$ for all $a, b, c \in A$. Since the range of $\Phi$ is dense, it follows that $\psi(u v, w)=\psi(u, v w)$ for all $u, v, w \in B$. 
Proposition 2.7. Let $A$ and $B$ be Banach algebras with the property $(\mathbb{B})$. Then the projective tensor product $A \otimes_{\pi} B$ has the property $(\mathbb{B})$.

Proof. Let $\phi:\left(A \otimes_{\pi} B\right) \times\left(A \otimes_{\pi} B\right) \rightarrow X$ be a continuous bilinear map preserving zero products, for some Banach space $X$. For $u, v \in B$ we define $\phi_{u, v}: A \times A \rightarrow X$ by $\phi_{u, v}(a, b)=\phi(a \otimes u, b \otimes v)$ for all $a, b \in A$. Then $\phi_{u, v}$ preserves zero products and so $\phi_{u, v}(a b, c)=\phi_{u, v}(a, b c)$ for all $a, b, c \in A$. We thus get

$$
\phi(a b \otimes u, c \otimes v)=\phi(a \otimes u, b c \otimes v) \quad(a, b, c \in A, u, v \in B) .
$$

Likewise we show that

$$
\phi(a \otimes u v, b \otimes w)=\phi(a \otimes u, b \otimes v w) \quad(a, b \in A, u, v, w \in B) .
$$

If $a, b, c \in A$ and $u, v, w \in B$, then we deduce from (2.2) and (2.3) that

$$
\begin{aligned}
\phi((a \otimes u)(b \otimes v), c \otimes w) & =\phi(a b \otimes u v, c \otimes w) \\
& =\phi(a \otimes u v, b c \otimes w)=\phi(a \otimes u, b c \otimes v w) \\
& =\phi(a \otimes u,(b \otimes v)(c \otimes w)) .
\end{aligned}
$$

Since the linear span of the elementary tensors $a \otimes u(a \in A, u \in B)$ is dense in $A \otimes_{\pi} B$ and $\phi$ is continuous, (2.4) implies that $\phi$ satisfies $\phi(x y, z)=$ $\phi(x, y z)$ for all $x, y, z \in A \otimes_{\pi} B$.

Let $\Omega$ be a locally compact Hausdorff space. We denote by $C_{0}(\Omega, A)$ the Banach algebra of all continuous functions from $\Omega$ into a Banach algebra $A$ which vanish at infinity.

Proposition 2.8. Let $\Omega$ be a locally compact Hausdorff space and let $A$ be a Banach algebra with the property $(\mathbb{B})$. Then $C_{0}(\Omega, A)$ has the prop$\operatorname{erty}(\mathbb{B})$.

Proof. It is well known that the map $f \otimes a \mapsto f(\cdot) a$ induces an isometric algebra isomorphism from the injective tensor product $C_{0}(\Omega) \otimes_{\epsilon} A$ onto $C_{0}(\Omega, A)$. We then consider the canonical map $\kappa: C_{0}(\Omega) \otimes_{\pi} A \rightarrow C_{0}(\Omega) \otimes_{\epsilon} A$, which is a homomorphism with dense range. On the other hand, $C_{0}(\Omega)$ is a $C^{*}$-algebra and so it has the property $(\mathbb{A})$ (Example 1.3(iii)) and therefore the property $(\mathbb{B})$ (see Theorem 2.11 below). By Proposition 2.7, $C_{0}(\Omega) \otimes_{\pi} A$ has the property $(\mathbb{B})$. Proposition 2.6 now shows that $C_{0}(\Omega) \otimes_{\epsilon} A$ has the property $(\mathbb{B})$.

Let $\left(A_{i}\right)_{i \in I}$ be a family of Banach algebras, and take $1 \leq p \leq \infty$. Then we write $\ell^{p}\left(I, A_{i}\right)$ and $c_{0}\left(I, A_{i}\right)$ for the Banach algebras defined in [14, Example 2.1.18(iii)].

Proposition 2.9. Let $\left(A_{i}\right)_{i \in I}$ be a family of Banach algebras with the property $(\mathbb{B})$. Then $\ell^{p}\left(I, A_{i}\right)$ with $1 \leq p<\infty$ and $c_{0}\left(I, A_{i}\right)$ have the prop$\operatorname{erty}(\mathbb{B})$. 
Proof. Let $A$ denote either $\ell^{p}\left(I, A_{i}\right)$ or $c_{0}\left(I, A_{i}\right)$ and let $A_{0}$ be the subalgebra of $A$ consisting of the families $\left(a_{i}\right)_{i \in I}$ which are zero except at a finite number of elements of $I$. Observe that $A_{0}$ is dense in $A$. We also consider the natural embeddings $\epsilon_{i}: A_{i} \rightarrow A$ with $i \in I$.

Let $\phi: A \times A \rightarrow X$ be a continuous bilinear map preserving zero products. For each $i \in I$, consider the map $\phi_{i}: A_{i} \times A_{i} \rightarrow X$ given by $\phi_{i}\left(a_{i}, b_{i}\right)=$ $\phi\left(\epsilon_{i}\left(a_{i}\right), \epsilon_{i}\left(b_{i}\right)\right)$ for all $a_{i}, b_{i} \in A_{i}$. It is clear that $\phi_{i}$ preserves zero products and so $\phi\left(\epsilon_{i}\left(a_{i} b_{i}\right), \epsilon_{i}\left(c_{i}\right)\right)=\phi\left(\epsilon_{i}\left(a_{i}\right), \epsilon_{i}\left(b_{i} c_{i}\right)\right)$ for all $a_{i}, b_{i}, c_{i} \in A_{i}$. This clearly entails that $\phi(a b, c)=\phi(a, b c)$ for all $a, b, c \in A_{0}$. Since $A_{0}$ is dense in $A$ and $\phi$ is continuous we finally arrive at $\phi(a b, c)=\phi(a, b c)$ for all $a, b, c \in A$.

2.3. Banach algebras with the property $(\mathbb{A})$ have the property $(\mathbb{B})$. The key tool for proving our fundamental result that the property $(\mathbb{A})$ implies the property $(\mathbb{B})$ is the following lemma concerning $\ell^{1}(\mathbb{Z})$. In our recent paper a similar result was obtained for the algebra $C(I)$ of continuous functions on a compact interval $I$ [2, Lemma 2.1]. In the following, $\mathcal{F}$ denotes the Fourier transform and $\mathbb{T}$ stands for the circle group.

Lemma 2.10. Let $\phi: \ell^{1}(\mathbb{Z}) \times \ell^{1}(\mathbb{Z}) \rightarrow X$ be a continuous bilinear map with the property

$$
a, b \in \ell^{1}(\mathbb{Z}), \operatorname{supp}(\mathcal{F}(a)) \cap \operatorname{supp}(\mathcal{F}(b))=\emptyset \Rightarrow \phi(a, b)=0 .
$$

Then $\phi$ is symmetric, i.e. $\phi(a, b)=\phi(b, a)$ for all $a, b \in \ell^{1}(\mathbb{Z})$.

Proof. Throughout the proof we shall consider the usual elements $\delta_{j}$ in $\ell^{1}(\mathbb{Z})$ defined by

$$
\delta_{j}(k)=\left\{\begin{array}{ll}
1, & j=k \\
0, & j \neq k
\end{array} \quad(j, k \in \mathbb{Z}) .\right.
$$

The map $\phi$ gives rise to a continuous linear operator $\Phi$ on the projective tensor product $\ell^{1}(\mathbb{Z}) \otimes_{\pi} \ell^{1}(\mathbb{Z})$ defined through

$$
\Phi(a \otimes b)=\phi(a, b) \quad\left(a, b \in \ell^{1}(\mathbb{Z})\right) .
$$

On the other hand, the Banach algebra $\ell^{1}(\mathbb{Z}) \otimes_{\pi} \ell^{1}(\mathbb{Z})$ may be identified with the Banach algebra $\ell^{1}(\mathbb{Z} \times \mathbb{Z})$ by defining

$$
(a \otimes b)(j, k)=a(j) b(k)
$$

for all $a, b \in \ell^{1}(\mathbb{Z})$ and $j, k \in \mathbb{Z}$. Hence $\Phi$ may be thought of as a continuous linear operator on $\ell^{1}(\mathbb{Z} \times \mathbb{Z})$.

Our first objective is to prove that $\Phi(x)=0$ whenever $x \in \ell^{1}(\mathbb{Z} \times \mathbb{Z})$ is such that

$$
\operatorname{supp}(\mathcal{F}(x)) \cap\{(z, z): z \in \mathbb{T}\}=\emptyset .
$$

Let $x \in \ell^{1}(\mathbb{Z} \times \mathbb{Z})$ satisfy (2.6), write $x=\sum_{n=1}^{\infty} u_{n} \otimes v_{n}$ with $u_{n}, v_{n} \in \ell^{1}(\mathbb{Z})$, let $\delta>0$ be such that

$$
\delta \leq|z-u|+|w-u| \quad((z, w) \in \operatorname{supp}(\mathcal{F}(x)), u \in \mathbb{T})
$$


and let $m \in \mathbb{N}$ be such that $8 \sin (\pi / m)<\delta$. We now consider the open subsets of $\mathbb{T}$ defined by

$$
U_{k}=\left\{z \in \mathbb{T}:\left|z-e^{2 \pi k / m i}\right|<2 \sin (\pi / m)\right\} \quad(k=1, \ldots, m) .
$$

Since $\bigcup_{k=1}^{m} U_{k}=\mathbb{T}$, it is well known that there exist smooth functions $\omega_{1}, \ldots, \omega_{m}$ on $\mathbb{T}$ with $\omega_{1}+\cdots+\omega_{m}=\mathbf{1}$ and $\operatorname{supp}\left(\omega_{k}\right) \subset U_{k}$ for $k=1, \ldots, m$. By the smoothness, we have $\omega_{k}=\mathcal{F}\left(a_{k}\right)$ for some $a_{k} \in \ell^{1}(\mathbb{Z})$ for each $k=1, \ldots, m$. We thus get elements $a_{1}, \ldots, a_{m} \in \ell^{1}(\mathbb{Z})$ such that

$$
a_{1}+\cdots+a_{m}=\delta_{0} \quad \text { and } \quad \operatorname{supp}\left(\mathcal{F}\left(a_{k}\right)\right) \subset U_{k} \quad(k=1, \ldots, m) .
$$

It is easily seen that

$$
\operatorname{supp}(\mathcal{F}(x)) \cap\left(U_{j} \times U_{k}\right)=\emptyset
$$

whenever $U_{j}$ and $U_{k}$ are such that $U_{j} \cap U_{k} \neq \emptyset$. This last property, together with the fact that $\sum_{k=1}^{m} a_{k}=\delta_{0}$, implies that

$$
\begin{aligned}
x & =\sum_{j=1}^{m} \sum_{k=1}^{m} x *\left(a_{j} \otimes a_{k}\right)=\sum_{U_{j} \cap U_{k}=\emptyset} x *\left(a_{j} \otimes a_{k}\right) \\
& =\sum_{U_{j} \cap U_{k}=\emptyset} \sum_{n=1}^{\infty}\left(u_{n} * a_{j}\right) \otimes\left(v_{n} * a_{k}\right) .
\end{aligned}
$$

Finally, we observe that

$$
\Phi(x)=\sum_{U_{j} \cap U_{k}=\emptyset} \sum_{n=1}^{\infty} \phi\left(u_{n} * a_{j}, v_{n} * a_{k}\right)=0,
$$

because of $(2.5)$ and

$$
\begin{aligned}
\operatorname{supp}\left(\mathcal{F}\left(u_{n} * a_{j}\right)\right) \cap \operatorname{supp}( & \left.\mathcal{F}\left(v_{n} * a_{k}\right)\right) \\
& =\operatorname{supp}\left(\mathcal{F}\left(u_{n}\right) \mathcal{F}\left(a_{j}\right)\right) \cap \operatorname{supp}\left(\mathcal{F}\left(v_{n}\right) \mathcal{F}\left(a_{k}\right)\right) \\
& \subset \operatorname{supp}\left(\mathcal{F}\left(a_{j}\right)\right) \cap \operatorname{supp}\left(\mathcal{F}\left(a_{k}\right)\right) \subset U_{j} \cap U_{k} .
\end{aligned}
$$

Our next goal is to show that $\Phi(x)=0$ whenever $x \in \ell^{1}(\mathbb{Z} \times \mathbb{Z})$ is such that

$$
\mathcal{F}(x)(z, z)=0 \quad \forall z \in \mathbb{T} .
$$

Let $x \in \ell^{1}(\mathbb{Z} \times \mathbb{Z})$ satisfy this condition. Since $\{(z, z): z \in \mathbb{T}\}$ is a set of synthesis for $\ell^{1}(\mathbb{Z} \times \mathbb{Z}$ ) (see [31, 7.5.1 and Theorem 7.5.2]), there exists a sequence $\left(x_{n}\right)$ in $\ell^{1}(\mathbb{Z} \times \mathbb{Z})$ such that $x_{n}$ satisfies (2.6) for each $n \in \mathbb{N}$ and $\lim x_{n}=x$. From what has previously been proved, it follows that $\Phi(x)=\lim \Phi\left(x_{n}\right)=0$.

We finally proceed to show that $\phi(a, b)=\phi(b, a)$ for all $a, b \in \ell^{1}(\mathbb{Z})$. Of course, we only need to consider the case where $a=\delta_{j}$ and $b=\delta_{k}$ for some $j, k \in \mathbb{Z}$. Set 


$$
x=\delta_{j} \otimes \delta_{k}-\delta_{k} \otimes \delta_{j}
$$

and observe that

$$
\mathcal{F}(x)(z, w)=z^{-j} w^{-k}-z^{-k} w^{-j} \quad(z, w \in \mathbb{T}),
$$

in particular, $\mathcal{F}(x)(z, z)=0$ for each $z \in \mathbb{T}$. This entails that $\Phi(x)=0$ and therefore $\phi\left(\delta_{j}, \delta_{k}\right)=\phi\left(\delta_{k}, \delta_{j}\right)$, as required.

Theorem 2.11. Let $A$ be a faithful Banach algebra, let $X$ be a Banach space, and let $\phi: A \times A \rightarrow X$ be a continuous bilinear map preserving zero products. Then

$$
\phi(a \mu, b)=\phi(a, \mu b)
$$

for all $a, b \in A$ and $\mu \in \overline{\mathcal{D}(A)}^{\mathrm{so}}$. In particular, if $A$ has the property $(\mathbb{A})$, then $A$ has the property $(\mathbb{B})$.

Proof. We begin by proving that

$$
\phi(a \mu, b)=\phi(a, \mu b)
$$

for all $a, b \in A$ and $\mu \in \mathcal{D}(A)$. Clearly it suffices to prove (2.8) for all doubly power-bounded elements $\mu$ in $\mathcal{M}(A)$.

Pick $a, b \in A$ and a doubly power-bounded element $\mu \in \mathcal{M}(A)$. We define a continuous linear operator

$$
\sigma_{\mu}: \ell^{1}(\mathbb{Z}) \rightarrow \mathcal{M}(A), \quad \sigma_{\mu}(\alpha)=\sum_{k=-\infty}^{\infty} \alpha(k) \mu^{k},
$$

for each $\alpha \in \ell^{1}(\mathbb{Z})$. We claim that

$$
\sigma_{\mu}(\alpha * \beta)=\sigma_{\mu}(\alpha) \sigma_{\mu}(\beta)
$$

for all $\alpha, \beta \in \ell^{1}(\mathbb{Z})$. Indeed,

$$
\begin{aligned}
\sigma_{\mu}(\alpha * \beta) & =\sum_{k=-\infty}^{\infty}(\alpha * \beta)(k) \mu^{k}=\sum_{k=-\infty}^{\infty}\left(\sum_{j=-\infty}^{\infty} \alpha(j) \beta(k-j)\right) \mu^{k} \\
& =\sum_{j=-\infty}^{\infty}\left(\alpha(j) \mu^{j}\left(\sum_{k=-\infty}^{\infty} \beta(k-j) \mu^{k-j}\right)\right) \\
& =\sum_{j=-\infty}^{\infty} \alpha(j) \mu^{j} \sigma_{\mu}(\beta)=\sigma_{\mu}(\alpha) \sigma_{\mu}(\beta) .
\end{aligned}
$$

We now consider the continuous bilinear map

$$
\psi: \ell^{1}(\mathbb{Z}) \times \ell^{1}(\mathbb{Z}) \rightarrow X, \quad \psi(\alpha, \beta)=\phi\left(a \sigma_{\mu}(\alpha), \sigma_{\mu}(\beta) b\right) \quad\left(\alpha, \beta \in \ell^{1}(\mathbb{Z})\right) .
$$

If $\alpha, \beta \in \ell^{1}(\mathbb{Z})$ are such that $\operatorname{supp}(\mathcal{F}(\alpha)) \cap \operatorname{supp}(\mathcal{F}(\beta))=\emptyset$, then $\alpha * \beta=0$ and, according to $(2.9)$, we have

$$
\left(a \sigma_{\mu}(\alpha)\right)\left(\sigma_{\mu}(\beta) b\right)=a \sigma_{\mu}(\alpha * \beta) b=0
$$


and so $\phi\left(a \sigma_{\mu}(\alpha), \sigma_{\mu}(\beta) b\right)=0$. This shows that $\psi$ satisfies (2.5). On account of Lemma 2.10, we have $\psi(\alpha, \beta)=\psi(\beta, \alpha)$ for all $\alpha, \beta \in \ell^{1}(\mathbb{Z})$. By taking $\alpha=e_{0}$ and $\beta=e_{1}$ we arrive at $\phi(a, \mu b)=\phi(a \mu, b)$, as required.

We now fix $a, b \in A$ and $\mu \in \overline{\mathcal{D}(A)}$ so. Let $\left(\mu_{i}\right)_{i \in I}$ be a net in $\mathcal{D}(A)$ converging to $\mu$ with respect to the strong operator topology on $\mathcal{M}(A)$. Since $\lim a \mu_{i}=a \mu$ and $\lim \mu_{i} b=\mu b$, the identity (2.8) together with the continuity of $\phi$ yields

$$
\phi(a \mu, b)=\lim _{i \in I} \phi\left(a \mu_{i}, b\right)=\lim _{i \in I} \phi\left(a, \mu_{i} b\right)=\phi(a, \mu b) .
$$

We now see that the class of Banach algebras with the property $(\mathbb{B})$ is really large, it contains algebras mentioned in Subsection 1.3, and has the extra advantage of being stable under standard constructions of Banach algebras.

\section{Linear maps preserving zero products}

Definition 3.1. Let $A$ and $B$ be Banach algebras and let $T: A \rightarrow B$ be a linear map. As mentioned in the introduction, $T$ is said to preserve zero products if

$$
a, b \in A, a b=0 \Rightarrow T(a) T(b)=0 .
$$

It is obvious that homomorphisms from $A$ into $B$ preserve zero products. The standard problem is to show that a map preserving zero products is "close" to a homomorphism. More precisely, one usually wants to describe $T$ as being a weighted homomorphism.

3.1. Weighted homomorphisms. Let $A$ be an algebra. A centralizer on $A$ is a linear operator $W: A \rightarrow A$ satisfying

$$
W(a b)=W(a) b=a W(b) \quad(a, b \in A) .
$$

The set $\Gamma(A)$ of all centralizers on $A$ is called the centroid of $A$. When endowed with the obvious operations, $\Gamma(A)$ becomes an algebra and the map $z \mapsto L_{z}\left(=R_{z}\right)$ gives an embedding of the centre $Z(A)$ of $A$ into $\Gamma(A)$. If $A$ is faithful, then the map $W \mapsto(W, W)$ yields an isomorphism from $\Gamma(A)$ onto $Z(\mathcal{M}(A))$, the centre of $\mathcal{M}(A)$. On the other hand, if $A$ is a faithful Banach algebra, then $\Gamma(A)$ is a closed subalgebra of $\mathcal{B}(A)$ and $W$ is injective whenever $W \in \Gamma(A)$ is such that $\overline{W(A)}=A$, which follows from $\operatorname{ker}(W) W(A)=W(A) \operatorname{ker}(W)=\{0\}$.

Definition 3.2. Let $A$ and $B$ be Banach algebras. As usual, a homomorphism from $A$ into $B$ is a linear map $\Phi: A \rightarrow B$ such that $\Phi(a b)=\Phi(a) \Phi(b)$ $(a, b \in A)$. We call a map $T: A \rightarrow B$ a weighted homomorphism if there exist a homomorphism $\Phi: A \rightarrow B$ and an invertible centralizer $W \in \Gamma(B)$ such that $T=W \Phi$. 
Note that any weighted homomorphism preserves zero products.

Proposition 3.3. Let $A$ be a Banach algebra with a left approximate identity $\left(\rho_{i}\right)_{i \in I}$, let $B$ be a faithful Banach algebra, and let $T: A \rightarrow B$ be a continuous weighted epimorphism. Then the decomposition $T=W \Phi$ with $W$ invertible in $\Gamma(B)$ and $\Phi: A \rightarrow B$ an epimorphism is unique and the following assertions are equivalent:

(i) $T$ is a homomorphism.

(ii) $\left(T\left(\rho_{i}\right)\right)_{i \in I}$ is a left approximate identity in $B$.

In particular, if $A$ has an identity, then $T$ is a homomorphism if and only if $T(\mathbf{1})=\mathbf{1}$.

Proof. Suppose that $T=U \Phi=V \Psi$ for some invertible centralizers $U, V \in \Gamma(B)$ and epimorphisms $\Phi, \Psi: A \rightarrow B$. Since $U$ and $V$ are necessarily continuous, so are $\Phi$ and $\Psi$. Moreover, $\Phi=W \Psi$, where $W=U^{-1} V \in \Gamma(B)$. We now observe that

$$
\begin{aligned}
W^{2}(\Psi(a b)) & =W(\Psi(a)) W(\Psi(b))=\Phi(a) \Phi(b) \\
& =\Phi(a b)=W(\Psi(a b)) \quad(a, b \in A) .
\end{aligned}
$$

By replacing $a$ by $\rho_{i}$ and taking limits we arrive at $W(\Psi(b))=W^{2}(\Psi(b))$ for each $b \in A$. Since $\Psi(A)=B$, it follows that $W=W^{2}$ and so $W$ is the identity operator.

Assume that $T$ is a homomorphism. If $u \in B$, then there is $a \in A$ with $T(a)=u$ and therefore $T\left(\rho_{i}\right) u=T\left(\rho_{i} a\right) \rightarrow T(a)$. Conversely, assume that $\left(T\left(\rho_{i}\right)\right)_{i \in I}$ is a left approximate identity for $B$. Since $\Phi$ is an epimorphism, $\left(\Phi\left(\rho_{i}\right)\right)_{i \in I}$ is a left approximate identity for $B$ and then, for each $u \in A$, we have

$$
u=\lim _{i \in I} T\left(\rho_{i}\right) u=\lim _{i \in I} W\left(\Phi\left(\rho_{i}\right)\right) u=\lim _{i \in I} W\left(\Phi\left(\rho_{i}\right) u\right)=W(u) .
$$

We thus get $T=\Phi$ and so $T$ is a homomorphism.

3.2. Characterizing homomorphisms through zero products

Lemma 3.4. Let $A$ and $B$ be Banach algebras, and let $T: A \rightarrow B$ be a continuous linear map preserving zero products. If $A$ has the property $(\mathbb{B})$, then

$$
T(a b) T(c)=T(a) T(b c) \quad(a, b, c \in A) .
$$

Accordingly, if $A$ and $B$ are unital and $T(\mathbf{1})=\mathbf{1}$, then $T$ is a homomorphism.

Proof. Define a continuous bilinear map $\phi: A \times A \rightarrow B$ by

$$
\phi(a, b)=T(a) T(b) \quad(a, b \in A) .
$$


Then $\phi(a, b)=0$ whenever $a, b \in A$ are such that $a b=0$, and so the property $(\mathbb{B})$ gives the required identity. If $A$ and $B$ are unital and $T(\mathbf{1})=\mathbf{1}$, then setting $c=\mathbf{1}$ in (3.1) we conclude that $T$ is a homomorphism.

Lemma 3.4 shows that the problem we are considering is trivial if $T(\mathbf{1})$ $=1$. However, group algebras rarely have an identity and therefore this assumption is too strong for us. Without this assumption the problem is challenging from the technical point of view and we require some information about partially defined multipliers.

Let $X$ and $Y$ be Banach spaces. A linear operator $T: \operatorname{dom}(T) \subset X \rightarrow Y$ is closed if its graph $\{(x, T(x)): x \in \operatorname{dom}(T)\}$ is closed in $X \times Y$. Of course, this property is equivalent to the following condition:

$$
\begin{aligned}
x_{n} \in \operatorname{dom}(T) \forall n \in \mathbb{N}, x_{n} \rightarrow x \in X, T\left(x_{n}\right) \rightarrow y \\
\quad \Rightarrow x \in \operatorname{dom}(T) \text { and } T(x)=y .
\end{aligned}
$$

The operator $T$ is closable if it has a closed extension, in which case the closure in $X \times Y$ of its graph is the graph of a linear operator $\bar{T}$, and that operator is the smallest closed extension of $T$, called the closure of $T$. It is readily seen that $T$ is closable if and only if the following property holds:

$$
x_{n} \in \operatorname{dom}(T) \forall n \in \mathbb{N}, x_{n} \rightarrow 0, T\left(x_{n}\right) \rightarrow y \Rightarrow y=0 .
$$

Lemma 3.5. Let $A$ be a left and right faithful Banach algebra, let $A_{U}$ and $A_{V}$ be dense subalgebras of $A$, and let $U: A_{U} \rightarrow A, V: A_{V} \rightarrow A$ be linear operators satisfying the conditions

$$
\begin{aligned}
& U(a b)=U(a) b \quad\left(a, b \in A_{U}\right), \\
& V(a b)=a V(b) \quad\left(a, b \in A_{V}\right), \\
& b U(a)=V(b) a \quad\left(a \in A_{U}, b \in A_{V}\right) .
\end{aligned}
$$

Then the operators $U$ and $V$ are closable, the domain $I_{\bar{U}}$ of $\bar{U}$ is a right ideal of $A$, the domain $I_{\bar{V}}$ of $\bar{V}$ is a left ideal of $A$, and the following identities hold:

$$
\begin{array}{ll}
\bar{U}(a b)=\bar{U}(a) b & \left(a \in I_{\bar{U}}, b \in A\right), \\
\bar{V}(a b)=a \bar{V}(b) & \left(a \in A, b \in I_{\bar{V}}\right), \\
b \bar{U}(a)=\bar{V}(b) a & \left(a \in I_{\bar{U}}, b \in I_{\bar{V}}\right) .
\end{array}
$$

Furthermore, if there exists a set $S \subset I_{U} \cap I_{V}$ which is dense in $A$ and such that $U(a)=V(a)$ for each $a \in S$, then both $I_{\bar{U}}$ and $I_{\bar{V}}$ are two-sided ideals of $A$ and

$$
\begin{array}{ll}
\bar{U}(b a)=b \bar{U}(a) & \left(a \in I_{\bar{U}}, b \in A\right), \\
\bar{V}(b a)=\bar{V}(b) a & \left(a \in A, b \in I_{\bar{V}}\right) .
\end{array}
$$


Proof. Let $\left(a_{n}\right)$ be a sequence in $A_{U}$ with $\lim a_{n}=0$ and $\lim U\left(a_{n}\right)=a$ for some $a \in A$. For every $b \in A_{V}$, we have

$$
b a=\lim b U\left(a_{n}\right)=\lim V(b) a_{n}=0 .
$$

Since $A_{V}$ is dense in $A$, it follows that $A a=\{0\}$ and therefore $a=0$. In the same way we check that $V$ is also closable.

Let $\bar{U}: I_{\bar{U}} \rightarrow A$ and $\bar{V}: I_{\bar{V}} \rightarrow A$ be the corresponding closures. Let $a \in I_{\bar{U}}, b \in I_{\bar{V}}$, and $c \in A$. Then there exist sequences $\left(a_{n}\right),\left(c_{n}\right)$ in $I_{U}$ and $\left(b_{n}\right),\left(d_{n}\right)$ in $I_{V}$ such that $\lim a_{n}=a, \lim U\left(a_{n}\right)=\bar{U}(a), \lim b_{n}=b$, $\lim V\left(b_{n}\right)=\bar{V}(b)$, and $\lim c_{n}=\lim d_{n}=c$. We now observe that $\left(a_{n} c_{n}\right)$ and $\left(d_{n} b_{n}\right)$ are sequences in $I_{U}$ and $I_{V}$, respectively, with

$$
U\left(a_{n} c_{n}\right)=U\left(a_{n}\right) c_{n} \rightarrow \bar{U}(a) c \quad \text { and } \quad V\left(d_{n} b_{n}\right)=d_{n} V\left(b_{n}\right) \rightarrow c \bar{V}(b) .
$$

This shows that $a c \in I_{\bar{U}}$ with $\bar{U}(a c)=\bar{U}(a) c$ and $c b \in I_{\bar{V}}$ with $\bar{V}(c b)=$ $c \bar{V}(b)$. On the other hand,

$$
b \bar{U}(a)=\lim b_{n} U\left(a_{n}\right)=\lim V\left(b_{n}\right) a_{n}=\bar{V}(b) a,
$$

as required.

Let $S$ satisfy the condition in the lemma. Let $a \in I_{\bar{U}}$ and $b \in A$. We take a sequence $\left(b_{n}\right)$ in $S$ with $\lim b_{n}=b$. The sequence $\left(b_{n} a\right)$ lies in $I_{\bar{U}}$, $\lim b_{n} a=b a$, and

$$
\bar{U}\left(b_{n} a\right)=\bar{U}\left(b_{n}\right) a=U\left(b_{n}\right) a=V\left(b_{n}\right) a=\bar{V}\left(b_{n}\right) a=b_{n} \bar{U}(a) \rightarrow b \bar{U}(a) .
$$

Since $\bar{U}$ is closed, the preceding property implies that $b a \in I_{\bar{U}}$ and $\bar{U}(b a)=$ $b \bar{U}(a)$. In the same way we check the corresponding property for $\bar{V}$.

Theorem 3.6. Let $A$ be a Banach algebra with the property $(\mathbb{B})$ and having a left approximate identity, and let $T: A \rightarrow B$ be a continuous linear map on some Banach algebra $B$ preserving zero products. Then there exist a right ideal $I_{V}$ of $B$ containing $T\left(A^{2}\right)$ and a linear operator $V: I_{V} \rightarrow B$ such that

$$
\begin{aligned}
V(u v)=V(u) v, \quad V(z w) & =z V(w) \\
(u & \left.\in I_{V}, v \in B, w \in \operatorname{alg} T\left(A^{2}\right), z \in \operatorname{alg} T(A)\right)
\end{aligned}
$$

and

$$
V(T(a b))=T(a) T(b) \quad(a, b \in A) .
$$

Furthermore, the following assertions hold:

(i) If $B$ is faithful and $\overline{T(A)}=B$, then there exist a two-sided ideal $I_{W}$ of $B$ containing $T\left(A^{2}\right)$ and a closed linear operator $W: I_{W} \rightarrow B$ such that

$$
W(u v)=W(u) v \quad \text { and } \quad W(v u)=v W(u) \quad\left(u \in I_{W}, v \in B\right)
$$


and

$$
W(T(a b))=T(a) T(b) \quad(a, b \in A) .
$$

Moreover, if $B$ is essential, then $\overline{W(B)}=B$ and $W$ is injective.

(ii) If $B$ is faithful and factors weakly, and $T(A)=B$, then the operator $W$ given in (i) is invertible and there exists a continuous epimorphism $\Phi: A \rightarrow I_{W}$ such that $T=W \Phi$.

Proof. Let $\left(\rho_{i}\right)_{i \in I}$ be a left approximate identity for $A$. Let $I_{V}=\{u \in B$ : $\left(T\left(\rho_{i}\right) u\right)_{i \in I}$ converges $\}$ and define $V: I_{V} \rightarrow B$ by

$$
V(u)=\lim _{i \in I} T\left(\rho_{i}\right) u \quad\left(u \in I_{V}\right) .
$$

It is clear that $I_{V}$ is a right ideal of $B$ and that

$$
V(u v)=V(u) v \quad\left(u \in I_{V}, v \in B\right) .
$$

By (3.1) we have

$$
T(a) T(b)=\lim _{i \in I} T\left(\rho_{i} a\right) T(b)=\lim _{i \in I} T\left(\rho_{i}\right) T(a b) \quad(a, b \in A) .
$$

This shows that $T\left(A^{2}\right) \subset I_{V}$ and

$$
V(T(a b))=T(a) T(b) \quad(a, b \in A) .
$$

On account of (3.1), we have $T(A) T\left(A^{2}\right)=T\left(A^{2}\right) T(A)$ so that $T(A) T\left(A^{2}\right)$ $\subset I_{V}$ and moreover

$$
\begin{aligned}
V\left(T\left(a_{1}\right) \cdots T\left(a_{n}\right) T(b c)\right) & =\lim _{i \in I} T\left(\rho_{i}\right) T\left(a_{1}\right) \cdots T\left(a_{n}\right) T(b c) \\
& =\lim _{i \in I} T\left(\rho_{i} a_{1}\right) \cdots T\left(a_{n}\right) T(b) T(c) \\
& =T\left(a_{1}\right) \cdots T\left(a_{n}\right) T(b) T(c) \\
& =T\left(a_{1}\right) \cdots T\left(a_{n}\right) V(T(b c))
\end{aligned}
$$

for all $a_{1}, \ldots, a_{n}, b, c \in A$. Consequently,

$$
V(z w)=z V(w) \quad\left(z \in \operatorname{alg} T(A), w \in \operatorname{alg} T\left(A^{2}\right)\right) .
$$

We now assume that $B$ is faithful and that $\overline{T(A)}=B$. Since $A$ has a left approximate identity, it follows that $A^{2}$ is dense in $A$, and since $T(A)$ is dense in $B$, so is $\operatorname{alg} T\left(A^{2}\right)$. On account of (3.2), (3.5) and Lemma 3.5, the restriction of the operator $V$ to $\operatorname{alg} T\left(A^{2}\right)$ is closable and the closure $W: I_{W} \rightarrow B$ of that operator has the properties given in (i). Moreover, from (3.4) we deduce that $T(A)^{2} \subset W\left(T\left(A^{2}\right)\right) \subset W(B)$ and so $\overline{B^{2}} \subset \overline{W(B)}$. This shows that $\overline{W(B)}=B$ in the case where $B$ is essential.

We now assume that $B$ factors weakly and $T(A)=B$. Then (3.4) clearly entails that $W$ is surjective. If, in addition, $B$ is faithful, then $W$ is injective. Indeed, for every $u \in \operatorname{ker} W$ and $v \in I_{W}$ we have

$$
u W(v)=W(u v)=W(u) v=0
$$


and similarly $W(v) u=0$. Since $W\left(I_{W}\right)=B$ and $B$ is faithful, it follows that $u=0$. It is straightforward to check that $W^{-1} \in \Gamma(B)$, which in particular entails that $W^{-1}$ is continuous. We now define $\Phi=W^{-1} T$. Of course, $\Phi$ is surjective and continuous. We proceed to show that $\Phi$ is a homomorphism. Let $a, b \in A$. On account of $W(T(a b))=T(a) T(b)$, we have

$$
\begin{aligned}
\Phi(a b) & =W^{-1}(T(a b))=W^{-1}\left(W^{-1}(T(a) T(b))\right) \\
& =W^{-1}\left(T(a) W^{-1}(T(b))\right)=W^{-1}(T(a) \Phi(b)) \\
& =W^{-1}(T(a)) \Phi(b)=\Phi(a) \Phi(b) .
\end{aligned}
$$

Let $A$ be a semiprime algebra, i.e. $I^{2} \neq\{0\}$ whenever $I$ is a nonzero two-sided ideal of $A$; this is equivalent to the condition that $a A a=\{0\}$, where $a \in A$, implies $a=0$, so in particular $A$ is both left and right faithful. An essentially defined centralizer on $A$ is a linear map $V: I_{V} \rightarrow A$ whose domain is a two-sided ideal $I_{V}$ of $A$ with $I_{V} \cap J \neq\{0\}$ for every nonzero two-sided ideal $J$ of $A$, and satisfying the following identities:

$$
V(a b)=V(a) b \quad \text { and } \quad V(b a)=b V(a) \quad(a \in I, b \in A) .
$$

Of course, every centralizer is an essentially defined centralizer. The relation $\simeq$ defined on the set $\mathcal{C}(A)$ of all essentially defined centralizers on $A$ by $U \simeq V$ if and only if there exists $W \in \mathcal{C}(A)$ such that both $U$ and $V$ extend $W$, is an equivalence relation. The set $C(A)$ of all equivalence classes under the obvious operations of partially defined operators is called the extended centroid of $A$, and was introduced by W. S. Martindale $[27,28]$ in the case where $A$ is a prime algebra, i.e. $I J \neq 0$ whenever $I$ and $J$ are nonzero two-sided ideals of $A$. In that case $C(A)$ is a field extension of the base field. It is interesting to note that $C(A)$ can be identified with the centre of the symmetric algebra of quotients of $A$ : the definition is very similar to that of $\mathcal{M}(A)$, operating with (equivalence classes of) partially defined multipliers.

A prime algebra $A$ is said to be centrally closed if the canonical embedding of the base field $\mathbb{F}$ into $C(A)$ is surjective. This means that for every partially defined centralizer $W: I \rightarrow A$ there is $\lambda \in \mathbb{F}$ such that $W(a)=\lambda a$ for each $a \in A$. Every primitive Banach algebra and every prime $C^{*}$-algebra is centrally closed. The first example follows from $[27,28]$ and the standard theory of Banach algebras, while the second one follows from [29].

Corollary 3.7. Let $A$ be a Banach algebra with a left approximate identity and the property $(\mathbb{B})$, let $B$ be a centrally closed prime Banach algebra, and let $T: A \rightarrow B$ be a continuous linear map preserving zero products. If $\overline{T(A)}=B$, then there exist a nonzero complex number $\lambda$ and a continuous homomorphism $\Phi: A \rightarrow B$ such that $T=\lambda \Phi$. Accordingly, $\overline{\Phi(A)}=B, \Phi$ is an epimorphism if $T(A)=B$, and $\Phi$ is an isomorphism if $T$ is bijective. 
Proof. Let $W$ be the partially defined centralizer given in assertion (i) of Theorem 3.6. Since $B$ is centrally closed, there exists a complex number $\alpha$ such that $W(u)=\alpha u$ for each $u \in I_{W}$. Hence $\alpha T(a b)=T(a) T(b)$ for all $a, b \in A$. This clearly forces that $\alpha \neq 0$ and that $\Phi=\alpha^{-1} T$ is a homomorphism.

It is worth pointing out that primitive Banach algebras and prime $C^{*}$ algebras are centrally closed (see Section 2), so Corollary 3.7 covers some interesting situations.

Roughly speaking, Theorem 3.6 shows that $T$ is "almost" a weighted homomorphism. When the left approximate identity is bounded, the conclusion of Theorem 3.6 can be strengthened, and in particular it can be shown that under suitable additional assumptions $T$ is actually a weighted homomorphism.

TheOREM 3.8. Let $A$ be a Banach algebra with the property $(\mathbb{B})$ and having a bounded left approximate identity, and let $T: A \rightarrow B$ be a continuous linear map on some Banach algebra $B$ preserving zero products. Then there exist a closed right ideal $I_{V}$ of $B$ containing $T(A)$ and a continuous linear operator $V: I_{V} \rightarrow B$ such that

$$
\begin{gathered}
\|V\| \leq\|T\| \sup _{i \in I}\left\|\rho_{i}\right\|, \\
V(u v)=V(u) v, \quad V(z w)=z V(w) \quad\left(u \in I_{V}, v \in B, w, z \in \overline{\operatorname{alg} T(A)}\right),
\end{gathered}
$$

and

$$
V(T(a b))=T(a) T(b) \quad(a, b \in A) .
$$

Furthermore, the following assertions hold:

(i) If $\overline{T(A)}=B$, then there exists $W \in \Gamma(B)$ such that

$$
\|W\| \leq\|T\| \sup _{i \in I}\left\|\rho_{i}\right\| \quad \text { and } \quad W(T(a b))=T(a) T(b) \quad(a, b \in A) .
$$

If $B$ is essential, then $\overline{W(B)}=B$. Moreover, if $B$ is essential and faithful, then $W$ is injective.

(ii) If $B$ is faithful and factors weakly, and $T(A)=B$, then the operator $W$ given in assertion (i) is invertible and there exists a continuous epimorphism $\Phi: A \rightarrow B$ such that $T=W \Phi$. Moreover, if $T$ is bijective, then $\Phi$ is an isomorphism and

$$
\left\|\Phi^{-1}\right\| \leq\|T\|\left\|T^{-1}\right\| \sup _{i \in I}\left\|\rho_{i}\right\| .
$$

Proof. We define $I_{V}$ and $V$ just as in the proof of Theorem 3.6. The boundedness of the net $\left(T\left(\rho_{i}\right)\right)_{i \in I}$ clearly implies that $I_{V}$ is closed. Since $A$ has a bounded left approximate identity, it follows that $A^{2}=A$ so that 
$T(A)=T\left(A^{2}\right) \subset I_{V}$ and

$$
V(z w)=z V(w) \quad(w, z \in \overline{\operatorname{alg} T(A)}) .
$$

On the other hand,

$$
\left\|T\left(\rho_{i}\right) u\right\| \leq \sup _{i \in I}\left\|\rho_{i}\right\|\|T\|\|u\| \quad(i \in I)
$$

and so $\|V(a)\| \leq \sup _{i \in I}\left\|\rho_{i}\right\|\|T\|\|a\|$, which shows that the operator $V$ is continuous with $\|V\| \leq\|T\| \sup _{i \in I}\left\|\rho_{i}\right\|$.

We now assume that $\overline{T(A)}=B$. Since $T(A) \subset I_{V}$ and $I_{V}$ is closed, it follows that $V$ is defined on $B$. Since $V$ is continuous, it follows that the operator $W$ given in Theorem 3.6 is nothing other than $V$.

A rough summary of Theorem 3.8 can be given as follows.

Corollary 3.9. Let $A$ be a Banach algebra with the property $(\mathbb{B})$ and having a bounded left approximate identity, and let $B$ be a Banach algebra which is faithful and factors weakly. If $T: A \rightarrow B$ is a continuous surjective linear map preserving zero products, then $T$ is a weighted epimorphism.

In order to apply the preceding results to group algebras, recall that $\Gamma\left(L^{1}(G)\right)=Z(M(G))$ for any locally compact group $G$. Consequently, we arrive at the following result.

Corollary 3.10. Let $G_{1}$ and $G_{2}$ be locally compact groups. If $T: L^{1}\left(G_{1}\right) \rightarrow L^{1}\left(G_{2}\right)$ is a continuous surjective linear map preserving zero products, then there are an invertible measure $\mu$ in the centre of the measure algebra $M\left(G_{2}\right)$ and a continuous epimorphism $\Phi: L^{1}\left(G_{1}\right) \rightarrow L^{1}\left(G_{2}\right)$ with $T=\mu \Phi$.

On the other hand, the information about $\left\|\Phi^{-1}\right\|$ given in assertion (ii) of Theorem 3.8 is very useful in the case of group algebras. G. V. Wood proved in [36] that if $G_{1}$ and $G_{2}$ are locally compact groups, and if $\Phi: L^{1}\left(G_{1}\right) \rightarrow$ $L^{1}\left(G_{2}\right)$ is an isomorphism with $\|\Phi\| \leq \frac{1}{2}(1+\sqrt{3})$, then $G_{1}$ and $G_{2}$ are isomorphic. In a joint paper with N. J. Kalton [24] they showed that when the groups are abelian or connected, the result still holds true when $\|\Phi\|<$ $\sqrt{2}$. According to Theorem 3.8 , if $G_{1}$ and $G_{2}$ are locally compact groups with the property that there exists a continuous bijective map $T: L^{1}\left(G_{1}\right) \rightarrow$ $L^{1}\left(G_{2}\right)$ preserving zero products and $\|T\|\left\|T^{-1}\right\| \leq \frac{1}{2}(1+\sqrt{3})$, then $G_{1}$ and $G_{2}$ are isomorphic.

J. J. Font and S. Hernández proved in [17] that if $G_{1}$ and $G_{2}$ are locally compact abelian groups, then every bijective linear map $T: L^{1}\left(G_{1}\right) \rightarrow$ $L^{1}\left(G_{2}\right)$ preserving zero products is automatically continuous and can be described as a weighted homomorphism. 
4. Characterizing derivations through zero products. Let $A$ be a Banach algebra and let $X$ be a Banach $A$-bimodule. As usual, a derivation from $A$ into $X$ is a linear operator $D: A \rightarrow X$ such that

$$
D(a b)=D(a) \cdot b+a \cdot D(b) \quad(a, b \in A) .
$$

Throughout this section we shall be concerned with the following properties of a continuous linear map $D: A \rightarrow X$ :

$$
\begin{aligned}
& a, b, c \in A, a b=b c=0 \Rightarrow a \cdot D(b) \cdot c=0 ; \\
& a, b \in A, a b=0 \Rightarrow D(a) \cdot b+a \cdot D(b)=0 .
\end{aligned}
$$

Our purpose is to investigate whether each of the above conditions characterizes derivations. In this regard we will arrive at a type of derivation-like operators, the so-called generalized derivations. They are closely related to the notion of a generalized derivation that first appeared in [7]. We point out that there are several papers which are concerned with the characterization of (generalized) derivations by their action on the zero product elements $[2,9,18,23,33,37]$.

4.1. Introducing generalized derivations. Let $A$ be a Banach algebra and let $X$ be a Banach $A$-bimodule. The bimodule $X$ is essential if $X=$ $\overline{\operatorname{lin}\{a \cdot x \cdot b: a, b \in A, x \in X\}}$. We write $Z_{A}(X)$ for the centre of $X$ :

$$
Z_{A}(X)=\{x \in X: a \cdot x=x \cdot a \forall a \in A\},
$$

and we define the annihilator of $A$ on $X$ by

$$
\operatorname{Ann}_{X}(S)=\{x \in X: a \cdot x \cdot b=0 \forall a, b \in S\} .
$$

Note that $X^{* *}$ is a Banach $A$-bimodule, and we may regard $X$ as a closed submodule of $X^{* *}$. In fact, the natural embedding $\iota_{X}: X \rightarrow X^{* *}$ is an $A$ bimodule homomorphism and so is the natural projection $\pi_{X}: X^{* * *} \rightarrow X^{*}$, which is nothing other than the dual operator $\iota_{X}^{*}$ of $\iota_{X}$.

Definition 4.1. Let $A$ be a Banach algebra and let $X$ be a Banach $A$ bimodule. A linear operator $D: A \rightarrow X$ is said to be a generalized derivation if there exists $\xi \in X^{* *}$ such that

$$
D(a b)=D(a) \cdot b+a \cdot D(b)-a \cdot \xi \cdot b \quad(a, b \in A) ;
$$

Generalized derivations from a Banach algebra into itself appear in [22, 23,37 .

It should be noted that if $D$ is a generalized derivation, then the map

$$
d: A \rightarrow X^{* *}, \quad d(a)=D(a)-a \cdot \xi \quad(a \in A)
$$

is a derivation. Accordingly, we can write $D=d+R_{\xi}$, where $R_{\xi}: A \rightarrow X^{* *}$ is defined by $R_{\xi}(a)=a \cdot \xi$ for each $a \in A$. It is interesting to note that we can also write $D=d^{\prime}+L_{\xi}$ for some derivation $d^{\prime}: A \rightarrow X^{* *}$, where $L_{\xi}: A \rightarrow X^{* *}$ is now defined by $L_{\xi}(a)=\xi \cdot a(a \in A)$. In fact, we have $R_{\xi}=\operatorname{ad}_{\xi}+L_{\xi}$, 
where $\operatorname{ad}_{\xi}: A \rightarrow X^{* *}$ is the derivation defined by $\operatorname{ad}_{\xi}(a)=a \cdot \xi-\xi \cdot a$ for each $a \in A$.

In some situations the element $\xi$ defining a generalized derivation can be chosen in the given module instead of requiring its second dual, in which case the corresponding derivation maps into $X$.

Proposition 4.2. Let $A$ be a Banach algebra with identity, and let $D: A \rightarrow X$ be a generalized derivation on some Banach A-bimodule $X$. Then there exists an element $\xi \in X$ such that

$$
D(a b)=D(a) b+a D(b)-a \cdot \xi \cdot b \quad(a, b \in A)
$$

and so there is a decomposition $D=d+R_{\xi}$ for some derivation $d: A \rightarrow X$. Furthermore, the following assertions are equivalent:

(a) $D$ is a derivation.

(b) $\mathbf{1} \cdot D(\mathbf{1}) \cdot \mathbf{1}=0$.

Proof. According to the definition, there exists $\zeta \in X^{* *}$ such that

$$
D(a b)=D(a) \cdot b+a \cdot D(b)-a \cdot \zeta \cdot b \quad(a, b \in A) .
$$

Note that this, in particular, entails that $a \cdot \zeta \cdot b \in X$ for all $a, b \in A$. Define $\xi=\mathbf{1} \cdot \zeta \cdot \mathbf{1} \in X$. It is clear that (4.1) holds with $\zeta$ replaced by $\xi$. On account of (4.1), we have

$$
\xi=D(\mathbf{1}) \cdot \mathbf{1}+\mathbf{1} \cdot D(\mathbf{1})-D(\mathbf{1}) .
$$

Since obviously $\xi=\mathbf{1} \cdot \xi \cdot \mathbf{1}$, it follows from $(4.2)$ that $\xi=\mathbf{1} \cdot D(\mathbf{1}) \cdot \mathbf{1}$. Therefore (4.1) can be written as

$$
D(a b)=D(a) \cdot b+a \cdot D(b)-a \cdot D(\mathbf{1}) \cdot b \quad(a, b \in A) .
$$

We now observe that if $D$ is a derivation, then $D(\mathbf{1})=D(\mathbf{1 1})=D(\mathbf{1}) \cdot \mathbf{1}$ $+\mathbf{1} \cdot D(\mathbf{1})$ and so $\mathbf{1} \cdot D(\mathbf{1}) \cdot \mathbf{1}=0$. On the other hand, from (4.3) we deduce that $D$ is a derivation when $\mathbf{1} \cdot D(\mathbf{1}) \cdot \mathbf{1}=0$.

Proposition 4.3. Let $A$ be a Banach algebra, and let $D: A \rightarrow X$ be a generalized derivation on some dual Banach A-bimodule $X$. Then there exists an element $\xi \in X$ such that

$$
D(a b)=D(a) b+a D(b)-a \cdot \xi \cdot b \quad(a, b \in A)
$$

and so there is a decomposition $D=d+R_{\xi}$ for some derivation $d: A \rightarrow X$. If $A$ has a bounded approximate identity $\left(\rho_{i}\right)_{i \in I}$ and $D$ is continuous, then the following assertions are equivalent:

(a) $D$ is a derivation.

(b) $\lim _{i \in I} a \cdot D\left(\rho_{i}\right) \cdot b=0$ for all $a, b \in A$.

If $\operatorname{Ann}_{X}(A)=\{0\}$, then (a) and (b) are also equivalent to

(c) $\sigma\left(X, X_{*}\right)-\lim _{i \in I} D\left(\rho_{i}\right)=0$, where $X_{*}$ is a predual A-bimodule of $X$. 
Proof. Pick $\zeta \in X^{* *}$ such that

$$
D(a b)=D(a) \cdot b+a \cdot D(b)-a \cdot \zeta \cdot b \quad(a, b \in A) .
$$

Let $X_{*}$ be a predual $A$-bimodule of $X$ and let $\pi_{X_{*}}: X^{* *} \rightarrow X$ be the natural projection. Set $\xi=\pi_{X_{*}}(\zeta) \in X$. By applying $\pi_{X_{*}}$ to (4.4), and taking into account that $\pi_{X_{*}}$ is an $A$-bimodule homomorphism and $\pi_{X_{*}}(X) \subset X$, we arrive at

$$
D(a b)=D(a) \cdot b+a \cdot D(b)-a \cdot \xi \cdot b \quad(a, b \in A) .
$$

Throughout the rest of the proof we assume that $A$ has a bounded approximate identity $\left(\rho_{i}\right)_{i \in I}$. Then we apply (4.5) to obtain

$$
\begin{aligned}
D(a) \cdot b & =\lim _{i \in I} D\left(a \rho_{i}\right) \cdot b=\lim _{i \in I}\left(D(a) \cdot \rho_{i} b+a \cdot D\left(\rho_{i}\right) \cdot b-a \xi \cdot \rho_{i} b\right) \\
& =D(a) \cdot b+\lim _{i \in I} a \cdot D\left(\rho_{i}\right) \cdot b-a \cdot \xi \cdot b
\end{aligned}
$$

and so

$$
\lim _{i \in I} a \cdot D\left(\rho_{i}\right) \cdot b=a \cdot \xi \cdot b \quad(a, b \in A) .
$$

The equivalence of (a) and (b) is now clear from (4.5) and (4.6).

We finally assume that $\operatorname{Ann}_{X}(A)=\{0\}$. We claim that $X_{*}$ is essential. On the contrary, suppose that $\overline{A \cdot X_{*} \cdot A} \neq X_{*}$. Then there exists $x \in X \backslash\{0\}$ such that $\left\langle a \cdot x_{*} \cdot b, x\right\rangle=0$ for all $a, b \in A$ and $x_{*} \in X_{*}$. Therefore $\left\langle x_{*}, b \cdot x \cdot a\right\rangle$ $=0$ for all $a, b \in A$ and $x_{*} \in X_{*}$ and so $b \cdot x \cdot a=0$ for all $a, b \in A$. Since $\operatorname{Ann}_{X}(A)=\{0\}$, we see that $x=0$, a contradiction.

If condition (b) holds and $x_{*} \in X_{*}$, then there are $a, b \in A$ and $y_{*} \in X_{*}$ such that $x_{*}=a \cdot y_{*} \cdot b$ and therefore

$$
\lim _{i \in I}\left\langle x_{*}, D\left(\rho_{i}\right)\right\rangle=\lim _{i \in I}\left\langle y_{*}, b \cdot D\left(\rho_{i}\right) \cdot a\right\rangle=0,
$$

which gives (c). Conversely, if (c) holds, then (4.6) yields

$$
\left\langle x_{*}, a \cdot \xi \cdot b\right\rangle=\lim _{i \in I}\left\langle x_{*}, a \cdot D\left(\rho_{i}\right) \cdot b\right\rangle=\lim _{i \in I}\left\langle b \cdot x_{*} \cdot a, D\left(\rho_{i}\right)\right\rangle=0
$$

for all $a, b \in A$ and $x_{*} \in X_{*}$. This implies that $a \cdot \xi \cdot b=0(a, b \in A)$ and (4.5) now shows that $D$ is a derivation.

Proposition 4.4. Let $A$ be a Banach algebra with a bounded approximate identity $\left(\rho_{i}\right)_{i \in I}$, and let $D: A \rightarrow X$ be a continuous generalized derivation on some Banach A-bimodule $X$. Then the following assertions are equivalent:

(a) $D$ is a derivation.

(b) $\lim _{i \in I} a \cdot D\left(\rho_{i}\right) \cdot b=0$ for all $a, b \in A$.

Proof. The proof can be done in more or less the same way as for the corresponding assertions in Proposition 4.3. 
4.2. Characterizing derivations through the condition (D1). The condition (D1) was considered before in $[2,18,33]$. The motivation for its study arises from the connection with the concept of a local derivation.

TheOREM 4.5. Let $A$ be a Banach algebra with the property $(\mathbb{B})$ and having a bounded approximate identity, let $X$ be a Banach A-bimodule such that $\operatorname{Ann}_{X}(A)=\{0\}$, and let $D: A \rightarrow X$ be a continuous linear operator satisfying

$$
a, b, c \in A, a b=b c=0 \Rightarrow a \cdot D(b) \cdot c=0 .
$$

Then $D$ is a generalized derivation, i.e. there exists $\xi \in X^{* *}$ such that

$$
D(a b)=D(a) \cdot b+a \cdot D(b)-a \cdot \xi \cdot b \quad(a, b \in A) .
$$

Furthermore, $\xi$ can be chosen in $X$ in each of the following cases:

(i) A has an identity.

(ii) $X$ is a dual A-bimodule.

Proof. We begin by proving that

$$
D(a b c)=D(a b) \cdot c+a \cdot D(b c)-a \cdot D(b) \cdot c \quad(a, b, c \in A) .
$$

Pick $a_{2}, b_{2} \in A$ with $a_{2} b_{2}=0$. We consider the bilinear map $\phi: A \times A \rightarrow X$ defined by

$$
\phi(a, b)=a \cdot D\left(b a_{2}\right) \cdot b_{2} \quad(a, b \in A) .
$$

On account of the given hypothesis on $D$, we see at once that $\phi$ preserves zero products. Consequently, the property $(\mathbb{B})$ yields

$$
a_{1} b_{1} \cdot D\left(c_{1} a_{2}\right) \cdot b_{2}=a_{1} \cdot D\left(b_{1} c_{1} a_{2}\right) \cdot b_{2} \quad\left(a_{1}, b_{1}, c_{1} \in A\right) .
$$

We now fix $a_{1}, b_{1}, c_{1} \in A$ and consider the bilinear map $\psi: A \times A \rightarrow X$ given by

$$
\psi(a, b)=a_{1} b_{1} \cdot D\left(c_{1} a\right) \cdot b-a_{1} \cdot D\left(b_{1} c_{1} a\right) \cdot b \quad(a, b \in A) .
$$

On account of (4.8), $\psi$ preserves zero products and therefore the property $(\mathbb{B})$ gives

$$
\begin{aligned}
a_{1} b_{1} \cdot D\left(c_{1} a_{2} b_{2}\right) & \cdot c_{2}-a_{1} \cdot D\left(b_{1} c_{1} a_{2} b_{2}\right) \cdot c_{2} \\
& +a_{1} \cdot D\left(b_{1} c_{1} a_{2}\right) \cdot b_{2} c_{2}-a_{1} b_{1} \cdot D\left(c_{1} a_{2}\right) \cdot b_{2} c_{2}=0
\end{aligned}
$$

for all $a_{1}, b_{1}, c_{1}, a_{2}, b_{2}, c_{2} \in A$. Note that (4.9) can be rewritten as

$$
c \cdot(a \cdot D(e b)-D(a e b)+D(a e) \cdot b-a \cdot D(e) \cdot b) \cdot d=0
$$

for all $a, b, c, d \in A$ and $e \in A^{2}$, which gives (4.7).

Let $\left(\rho_{i}\right)_{i \in I}$ be a bounded approximate identity of $A$. Since the net $\left(D\left(\rho_{i}\right)\right)_{i \in I}$ is bounded, we can assume that it converges to $\xi \in X^{* *}$ with respect to the topology $\sigma\left(X^{* *}, X^{*}\right)$.

On account of (4.7), for all $a, b \in A$ we have

$$
D\left(a \rho_{i} b\right)=D\left(a \rho_{i}\right) \cdot b+a \cdot D\left(\rho_{i} b\right)-a \cdot D\left(\rho_{i}\right) \cdot b,
$$


so that

$$
a \cdot D\left(\rho_{i}\right) \cdot b=D\left(a \rho_{i}\right) \cdot b+a \cdot D\left(\rho_{i} b\right)-D\left(a \rho_{i} b\right)
$$

and therefore the net $\left(a \cdot D\left(\rho_{i}\right) \cdot b\right)_{i \in I}$ converges to $D(a) \cdot b+a \cdot D(b)-D(a b)$ with respect to the norm topology. On the other hand, for each $x_{*} \in X_{*}$ we have

$$
\begin{aligned}
\left\langle x_{*}, \lim _{i \in I} a \cdot D\left(\rho_{i}\right) \cdot b\right\rangle & =\lim _{i \in I}\left\langle x_{*}, a \cdot D\left(\rho_{i}\right) \cdot b\right\rangle=\lim _{i \in I}\left\langle b \cdot x_{*} \cdot a, D\left(\rho_{i}\right)\right\rangle \\
& =\left\langle b \cdot x_{*} \cdot a, \xi\right\rangle=\left\langle x_{*}, a \cdot \xi \cdot b\right\rangle .
\end{aligned}
$$

We thus get

$$
D(a b)=D(a) \cdot b+a \cdot D(b)-a \cdot \xi \cdot b \quad(a, b \in A) .
$$

4.3. Characterizing derivations through the condition (D2). The condition (D2) has been considered in [9, 23, 37].

THEOREM 4.6. Let $A$ be a Banach algebra with the property $(\mathbb{B})$ and having a bounded approximate identity, let $X$ be a Banach A-bimodule such that $\operatorname{Ann}_{X}(A)=\{0\}$, and let $D: A \rightarrow X$ be a continuous linear operator satisfying

$$
a, b \in A, a b=0 \Rightarrow D(a) \cdot b+a \cdot D(b)=0 .
$$

Then $D$ is a generalized derivation and there exists $\xi \in Z_{A}\left(X^{* *}\right)$ such that

$$
D(a b)=D(a) \cdot b+a \cdot D(b)-a \cdot \xi \cdot b \quad(a, b \in A) .
$$

Accordingly, there exists a continuous derivation $d: A \rightarrow X^{* *}$ such that

$$
D(a)=d(a)+a \cdot \xi \quad(a \in A) .
$$

Furthermore, $\xi$ can be chosen in $Z_{A}(X)$ in each of the following cases:

(i) A has an identity.

(ii) $X$ is a dual A-bimodule.

Proof. Suppose that $a, b, c \in A$ are such that $a b=b c=0$. According to the hypothesis, we have

$$
0=(D(a) \cdot b+a \cdot D(b)) c=D(a) \cdot b c+a \cdot D(b) \cdot c=a \cdot D(b) \cdot c .
$$

Let $\xi \in X^{* *}$ be given by Theorem 4.5. If $a, b \in A$ are such that $a b=0$, then

$$
0=D(a b)=D(a) \cdot b+a \cdot D(b)-a \cdot \xi \cdot b=-a \cdot \xi \cdot b
$$

and therefore we can apply the property $(\mathbb{B})$ to the bilinear map $(a, b) \mapsto$ $a \cdot \xi \cdot b$. We thus get

$$
a b \cdot \xi \cdot c=a \cdot \xi \cdot b c \quad(a, b, c \in A) .
$$

In cases (i) and (ii) we can use the assumption $\operatorname{Ann}_{X}(A)=\{0\}$ to conclude from (4.10) that $b \cdot \xi=\xi \cdot b$ for each $b \in A$. 
We now turn to the general case. Let $\left(\rho_{i}\right)_{i \in I}$ be a bounded approximate identity in $A$. Since the net $\left(\rho_{i} \cdot \xi \cdot \rho_{i}\right)_{i \in I}$ is bounded, we can assume that it converges to $\zeta \in X^{* *}$ with respect to the topology $\sigma\left(X^{* *}, X^{*}\right)$. We claim that

$$
D(a b)=D(a) \cdot b+a \cdot D(b)-a \cdot \zeta \cdot b \quad(a, b \in A) .
$$

Of course, it suffices to prove that $a \cdot \zeta \cdot b=a \cdot \xi \cdot b$ for all $a, b \in A$. Pick $a, b \in A$. Then

$$
\begin{aligned}
\left\langle x^{*}, a \cdot \zeta \cdot b\right\rangle & =\left\langle b \cdot x^{*} \cdot a, \zeta\right\rangle=\lim _{i \in I}\left\langle b \cdot x^{*} \cdot a, \rho_{i} \cdot \xi \cdot \rho_{i}\right\rangle \\
& =\lim _{i \in I}\left\langle\rho_{i} b \cdot x^{*} \cdot a \rho_{i}, \xi\right\rangle=\left\langle b \cdot x^{*} \cdot a, \xi\right\rangle=\left\langle x^{*}, a \cdot \xi \cdot b\right\rangle
\end{aligned}
$$

for each $x^{*} \in X^{*}$.

We finally show that $\zeta \in Z_{A}\left(X^{* *}\right)$. Set $b \in A$. On account of (4.10), we have

$$
\begin{aligned}
\rho_{i}^{2} b \cdot\left(\rho_{i} \cdot \xi \cdot \rho_{i}\right) & =\rho_{i}\left(\rho_{i} b \rho_{i}\right) \cdot \xi \cdot \rho_{i}=\rho_{i} \cdot \xi \cdot\left(\rho_{i} b \rho_{i}\right) \rho_{i} \\
& =\left(\rho_{i} \cdot \xi \cdot \rho_{i}\right) \cdot b \rho_{i}^{2}
\end{aligned}
$$

for each $i \in I$. On the other hand, for every $x^{*} \in X^{*}$, we have

$$
\begin{aligned}
\left\langle x^{*}, \rho_{i}^{2} b \cdot\left(\rho_{i} \cdot \xi \cdot \rho_{i}\right)\right\rangle-\left\langle x^{*}, b \cdot \zeta\right\rangle & \\
& =\left\langle x^{*},\left(\rho_{i}^{2} b-b\right) \cdot\left(\rho_{i} \cdot \xi \cdot \rho_{i}\right)\right\rangle+\left\langle x^{*} \cdot b, \rho_{i} \cdot \xi \rho_{i}\right\rangle-\left\langle x^{*} \cdot b, \zeta\right\rangle .
\end{aligned}
$$

Since

$$
\begin{aligned}
\left|\left\langle x^{*},\left(\rho_{i}^{2} b-b\right) \cdot\left(\rho_{i} \cdot \xi \cdot \rho_{i}\right)\right\rangle\right| \leq\left\|x^{*}\right\|\left\|\rho_{i}^{2} b-b\right\|\left\|\rho_{i} \cdot \xi \cdot \rho_{i}\right\| \\
\leq\left\|x^{*}\right\|\left(\left\|\rho_{i}\right\|+1\right)\left\|\rho_{i} b-b\right\|\left\|\rho_{i}\right\|^{2} \sup _{\|u\|=\|v\|=1}\|u \cdot \xi \cdot v\| \rightarrow 0
\end{aligned}
$$

and

$$
\lim _{i \in I}\left(\left\langle x^{*} \cdot b, \rho_{i} \cdot \xi \rho_{i}\right\rangle-\left\langle x^{*} \cdot b, \zeta\right\rangle\right)=0
$$

it follows that

$$
\lim _{i \in I}\left\langle x^{*}, \rho_{i}^{2} b \cdot\left(\rho_{i} \cdot \xi \cdot \rho_{i}\right)\right\rangle=\left\langle x^{*}, b \cdot \zeta\right\rangle .
$$

In the same way we check that

$$
\lim _{i \in I}\left\langle x^{*},\left(\rho_{i} \cdot \xi \cdot \rho_{i}\right) \cdot b \rho_{i}^{2}\right\rangle=\left\langle x^{*}, \zeta \cdot b\right\rangle
$$

and therefore (4.12) gives $\left\langle x^{*}, b \cdot \zeta\right\rangle=\left\langle x^{*}, \zeta \cdot b\right\rangle$ for each $x^{*} \in X^{*}$ and so $b \cdot \zeta=\zeta \cdot b$, as claimed.

Acknowledgements. The first, the third and the fourth named authors were supported by MEC (Spain) Grant MTM2006-04837 and Junta de Andalucía Grants FQM-185 and Proyecto de Excelencia P06-FQM-01438. The second author was supported by ARRS Grant P1-0288. 


\section{References}

[1] Y. A. Abramovich and A. K. Kitover, Inverses of disjointness preserving operators, Mem. Amer. Math. Soc. 143 (2000), no. 679.

[2] J. Alaminos, M. Brešar, J. Extremera, and A. R. Villena, Characterizing homomorphisms and derivations on $C^{*}$-algebras, Proc. Roy. Soc. Edinburgh Sect. A 137 (2007), 1-7.

[3] J. Araujo and K. Jarosz, Biseparating maps between operator algebras, J. Math. Anal. Appl. 282 (2003), 48-55.

[4] S. Banach, Théorie des opérations linéaires, Warszawa, 1932.

[5] E. Beckenstein and L. Narici, The separating map: a survey, Rend. Circ. Mat. Palermo 52 (1998), 637-648.

[6] F. F. Bonsall and J. Duncan, Complete Normed Algebras, Ergeb. Math. Grenzgeb. 80, Springer, New York, 1973.

[7] M. Brešar, On the distance of the composition of two derivations to the generalized derivations, Glasgow Math. J. 33 (1991), 89-93.

[8] -, Characterizing homomorphisms, derivations and multipliers in rings with idempotents, Proc. Roy. Soc. Edinburgh Sect. A 137 (2007), 9-21.

[9] M. A. Chebotar, W.-F. Ke and P.-H. Lee, Maps characterized by action on zero products, Pacific J. Math. 216 (2004), 217-228.

[10] - - - - Maps preserving zero Jordan products on hermitian operators, Illinois J. Math. 49 (2006), 445-452.

[11] M. A. Chebotar, W.-F. Ke, P.-H. Lee and N.-C. Wong, Mappings preserving zero products, Studia Math. 155 (2003), 77-94.

[12] M. A. Chebotar, W.-F. Ke, P.-H. Lee and R. Zhang, On maps preserving zero Jordan products, Monatsh. Math. 149 (2006), 91-101.

[13] J. Cui and J. Hou, Linear maps on von Neumann algebras preserving zero products or tr-rank, Bull. Austral. Math. Soc. 65 (2002), 79-91.

[14] H. G. Dales, Banach Algebras and Automatic Continuity, London Math. Soc. Monogr. 24, Oxford Univ. Press, New York, 2000.

[15] R. S. Doran and J. Wichmann, Approximate Identities and Factorization in Banach Modules, Lecture Notes in Math. 768, Springer, Berlin, 1979.

[16] G. B. Folland, A Course in Abstract Harmonic Analysis, Stud. Adv. Math., CRC Press, Boca Raton, FL, 1995.

[17] J. J. Font and S. Hernández, Automatic continuity and representation of certain isomorphisms between group algebras, Indag. Math. (N.S.) 6 (1995), 397-409.

[18] D. Hadwin and J. Li, Local derivations and local automorphisms, J. Math. Anal. Appl. 290 (2004), 702-714.

[19] E. Hewitt and K. A. Ross, Abstract Harmonic Analysis. Vol. II: Structure and Analysis for Compact Groups. Analysis on Locally Compact Abelian Groups, Grundlehren Math. Wiss. 152, Springer, New York, 1970.

[20] J. Hou and L. Zhao, Zero-product preserving additive maps on symmetric operator spaces and self-adjoint operator spaces, Linear Algebra Appl. 399 (2005), 235-244.

[21] —, - Jordan zero-product preserving additive maps on operator algebras, J. Math. Anal. Appl. 314 (2006), 689-700.

[22] W. Jing and S. Lu, Topological reflexivity of the spaces of $(\alpha, \beta)$-derivations on operator algebras, Studia Math. 156 (2003), 121-131.

[23] W. Jing, S. Lu, and P. Li, Characterisations of derivations on some operator algebras, Bull. Austral. Math. Soc. 66 (2002), 227-232. 
[24] N. J. Kalton and G. V. Wood, Homomorphisms of group algebras with norm less than $\sqrt{2}$, Pacific J. Math. 62 (1976), 439-460.

[25] W.-F. Ke, B.-R. Li and N.-C. Wong, Zero product preserving maps of operator-valued functions, Proc. Amer. Math. Soc. 132 (2003), 1979-1985.

[26] J. Lamperti, On the isometries of certain function-spaces, Pacific J. Math. 8 (1958), 459-466.

[27] W. S. Martindale 3rd, Prime rings satisfying a generalized polynomial identity, J. Algebra 12 (1969), 574-584.

[28] -, Lie isomorphisms of prime rings, Trans. Amer. Math. Soc. 142 (1969), 437-455.

[29] M. Mathieu, Elementary operators on prime $C^{*}$-algebras. I, Math. Ann. 284 (1989), 223-244.

[30] L. Molnár, Selected Preserver Problems on Algebraic Structures of Linear Operators and on Function Spaces, Lecture Notes in Math. 1895, Springer, Berlin, 2007.

[31] W. Rudin, Fourier Analysis on Groups, Interscience Publ., New York, 1962.

[32] V. Runde, Lectures on Amenability, Lecture Notes in Math. 1774, Springer, Berlin, 2002 .

[33] J. Schweizer, An analogue of Peetre's theorem in noncommutative topology, Quart. J. Math. 52 (2001), 499-506.

[34] M. Takesaki, Theory of Operator Algebras. I, Springer, New York, 1979.

[35] M. Wolff, Disjointness preserving operators on $C^{*}$-algebras, Arch. Math. (Basel) 62 (1994), 248-253.

[36] G. V. Wood, Isomorphisms of group algebras, Bull. London Math. Soc. 15 (1983), $247-252$.

[37] J. Zhu and C. Xiong, Generalized derivable mappings at zero point on some reflexive operator algebras, Linear Algebra Appl. 397 (2005), 367-379.

Departamento de Análisis Matemático

Facultad de Ciencias

Universidad de Granada

18071 Granada, Spain

E-mail: alaminos@ugr.es

jlizana@ugr.es

avillena@ugr.es
Faculty of Mathematics and Physics University of Ljubljana 1000 Ljubljana, Slovenia and Faculty of Natural Sciences and Mathematics University of Maribor 2000 Maribor, Slovenia E-mail: bresar@uni-mb.si

Received September 2, 2008

Revised version January 2, 2009 